\title{
Childhood Central Nervous System Yolk
} Sac Tumor

National Cancer Institute

\section{Source}

National Cancer Institute. Childhood Central Nervous System Yolk Sac Tumor. NCI

Thesaurus. Code C6209.

A yolk sac tumor that arises from the central nervous system and occurs during

childhood. 